\title{
STATUS OF MOLECULAR OPACITIES OF INTEREST IN THE MODELING OF A PROTO-SOLAR NEBULA
}

\author{
W. F. HUEBNER and L. W. FULLERTON \\ Los Alamos Scientific Laboratory, Los Alamos, N.M., U.S.A.
}

\begin{abstract}
The report centers on general procedures applicable to the calculation of constitutive properties (equation of state and opacity) of media that serve as models for the solar nebula during planet formation and for the atmospheres of some planets. Specifically considered are the equilibrium compositions of a mixture of atoms, molecules, and their ionic species in the gaseous phase, condensation into grains with refractory cores and mantles of volatile compounds, and the 'optical' properties of the grain-gas medium. A summary of available and still needed basic (input) data and some currently available results are presented.
\end{abstract}

\section{Motivation for Opacity Calculations}

In atmospheres energy is transported by radiation and convection. In the radiative mode, transfer is determined by the coefficients for emission, absorption, and scattering; these, in turn, depend on the atomic and molecular structure and equation of state of the medium. General methods are reviewed to predict these properties at temperatures, densities, and compositions suitable for modeling conditions to be expected in a proto-solar nebula before and during the time of planet formation, in proto-planets, and in sun-lit atmospheres throughout the history of planets. Although local thermodynamic equilibrium (LTE) does not always apply it has been adopted as a simplifying assumption.

The energy transfer in the proto-solar nebula determines the rate of its cooling and thus affects its chemical composition and the chemical makeup of the planets (Larimer, 1967; Larimer and Anders, 1967; Studier et al., 1968; Hayatsu et al., 1968; Anders, 1968, 1971). The diffusion approximation, using an extinction coefficient averaged over all photon energies (i.e., the Rosseland opacity), can be applied to optically thick atmospheres. For optically thin atmospheres photon-group mean opacities (Rosseland or Planck) are required to solve the more complicated radiation transfer equation.

\section{Earlier Calculations}

Some of the earlier predictions of opacities used in models for different stages of evolution during planet formation were carried out by Hayatsu and Nakano (1965), Gaustad (1963), Auman and Bodenheimer (1967), and Cox and Stewart (1965). Each of these applied only to a particular phase: grains, molecules, ions, or a composite synthesized artificially and very approximately from these subresults.

A more satisfactory calculational procedure is one in which all stages are treated in one program permitting continuous transformations from one phase to the next with simultaneous coexistence of several phases, e.g., molecular absorption must still be 
considered after grains have formed and atomic absorption still contributes after molecules have formed.

\section{Continuity of Opacity During Phase Transformations}

The Rosseland mean opacity is defined by

$$
\kappa_{R} \equiv \frac{\int_{0}^{\infty} \partial B_{v}(T) / \partial T \mathrm{~d} v}{\int_{0}^{\infty} \kappa_{v}^{-1} \partial B_{v}(T) / \partial T \mathrm{~d} v}
$$

and the Planck mean opacity is

$$
\kappa_{P} \equiv \frac{\int_{0}^{\infty} \kappa_{v}^{(a)^{\prime}} B_{v}(T) \mathrm{d} v}{\int_{0}^{\infty} B_{v}(T) \mathrm{d} v},
$$

where the mass extinction coefficient depending on frequency, $\nu$, is

$$
\kappa_{v}=\kappa_{v}^{(a) \prime}+\kappa_{v}^{(s)}
$$

$\kappa_{v}^{(a)^{\prime}}$ is the mass absorption coefficient corrected for stimulated emission, $\kappa_{v}^{(s)}$ the mass scattering coefficient, and $B_{v}(T)$ is the Planck function at temperature $T$. The major differences between these mean opacities are: (1) in the Planck mean the large values of $\kappa_{v}^{(a)^{\prime}}$, i.e., the strong absorption lines, add most heavily, while in the Rosseland mean the low values of $\kappa_{v}$, i.e., the continuum extinction in the windows between lines contributes most; (2) the individual absorption processes, e.g., atomic and molecular line and continuum absorption and absorption by grains, are additive in the Planck mean, they do not add in the Rosseland mean; (3) the weighting functions for the Planck mean peaks at $u=h v / k T \approx 2.8$, while that for the Rosseland mean peaks at $u \approx 3.8$; (4) the Rosseland opacity includes scattering, the Planck opacity does not. One frequently overlooked, but very important property of the Rosseland mean at low temperatures is the required behavior of the absorption coefficient in the long wavelength limit. Dividing the range of integration in the denominator of Equation (1) into two regions: from 0 to $\nu_{1}$ and from $\nu_{1}$ to $\infty$ with the constraint that $h v_{1}<k T$ and expanding the exponentials occurring in the derivative of the Planck function in a polynomial, one obtains for the integral in the first region

$$
\int_{0}^{v_{1}} \frac{1}{\kappa_{v}} \frac{\partial B_{v}(T)}{\partial T} \mathrm{~d} v=\frac{2 k^{4} T^{3}}{c^{2} h^{3}} \int_{0}^{u_{1}} \frac{u^{2}}{\kappa_{u}}\left(1-\frac{u^{2}}{12}+\cdots\right) \mathrm{d} u .
$$

The usual procedure of writing the integral in terms of $u=h v / k T$ has been adopted (the functional form of $\kappa_{u}$ is the same as for $\kappa_{v}$ ) and $u_{1}<1$. If the leading term of a 
polynomial expansion of $\kappa_{u}$ is const $\times u^{\alpha}$ then $\alpha$ must be less than 3. For $\alpha \geqslant 3$ Equation (4) will diverge and the Rosseland opacity is zero independent of the behavior of the extinction coefficient for $u>0$. For Rayleigh scattering by atoms, molecules, and grains the cross section is proportional to $\lambda^{-4}$, i.e., $\kappa_{u} \sim u^{4}$. Hence Rayleigh scattering alone is insufficient to yield a non-zero Rosseland opacity and other absorption processes must be included even if they appear to have negligibly small cross sections. At temperatures sufficiently high for free electrons to exist, inverse bremsstrahlung provides the needed additional absorption. At low temperatures it can be provided by electrically conducting grains (non-zero imaginary part of the refractive index) or molecular absorption, i.e., by the vibration-rotation or pure rotational spectrum of molecules still in the gaseous phase.

Figure 1 illustrates the relationship of the weighting function of Equation (1) (the

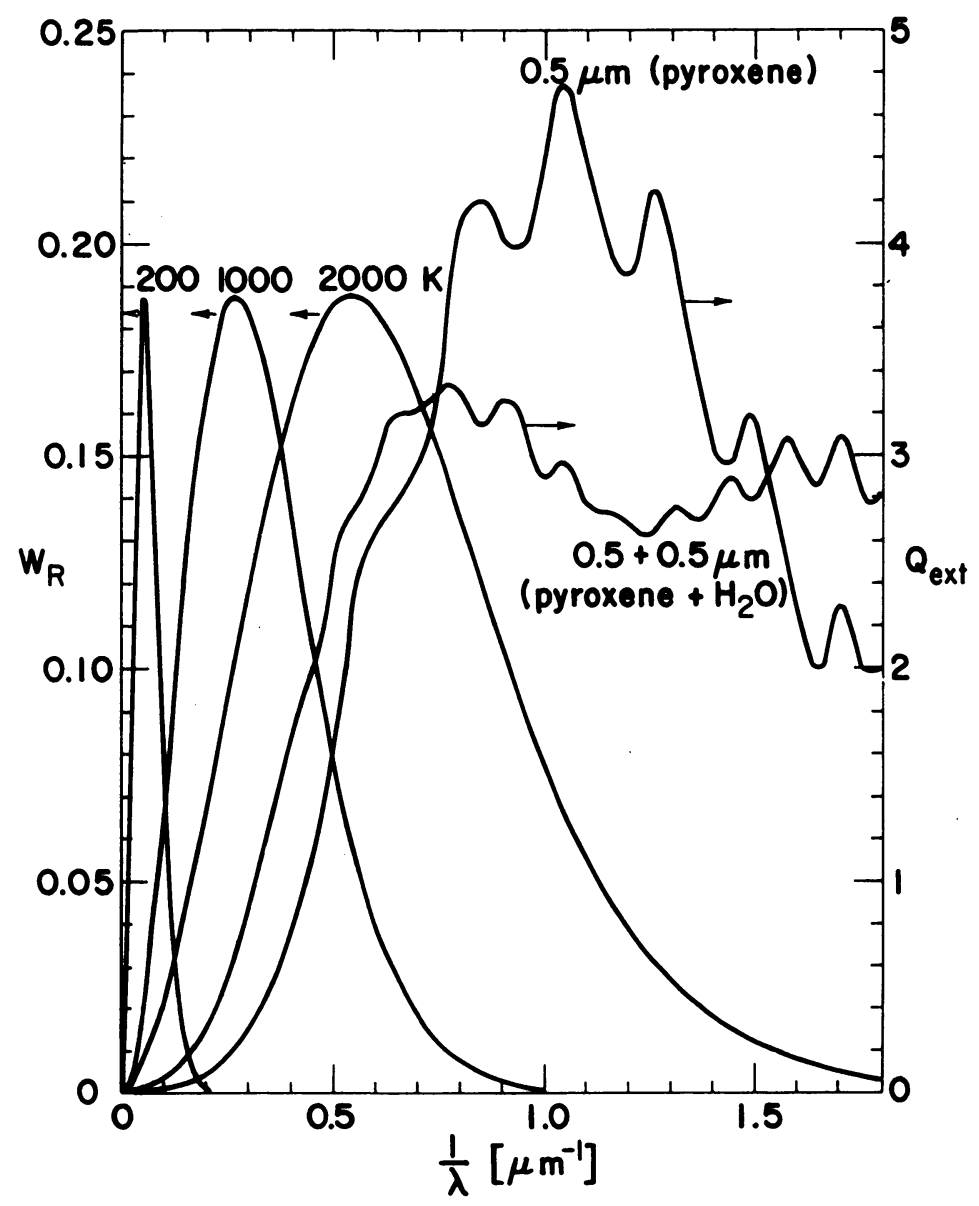

Fig. 1. Extinction efficiency, $Q_{\text {ext }}$, for pyroxene grain $(r=0.5 \mu \mathrm{m})$ and pyroxene with $\mathrm{H}_{2} \mathrm{O}$ mantle (scale on right) vs $1 / \lambda$ compared to Rosseland weighting functions, $W_{R}$, for three different temperatures (scale on left). 
Rosseland weighting function) to the extinction efficiency $Q_{\text {ext }}$ for a spherical $(r=0.5 \mu \mathrm{m})$ pyroxene, $(\mathrm{Mg}, \mathrm{Fe}) \mathrm{SiO}_{3}$, grain and a pyroxene grain covered with a $0.5 \mu \mathrm{m}$ thick ice-mantle. The refractive index for the ice was assumed constant and real, with $m=1.33$; for pyroxene data from Huffman and Stapp (1971) for frequency dependent real and imaginary parts were used. Their measurements indicate, however, that the imaginary part of the refractive index goes to zero as $1 / \lambda$ becomes small. Thus pyroxene and ice-covered pyroxene cross sections exhibit pure Rayleigh scattering at long wavelength. Another feature illustrated in Figure 1 is that the large extinction features of submicron grains are in the high photon energy tail of the Rosseland weighting function. For the Planck mean the weighting function is still smaller in the region where submicron grain absorption is large. Only small refractory grains will exist at temperatures of about 1000 to $2000 \mathrm{~K}$, above several thousand degrees all grains will have vaporized.

The vibration-rotation transitions in the infrared and the pure rotational transitions in the microwave region will be very important contributors to opacity - for the Rosseland mean because they prevent it from going to zero, for the Planck mean because of their relatively high absorption cross sections. There are about 600 known microwave transitions originating from 34 simple, volatile molecules composed of $\mathrm{H}-\mathrm{C}-\mathrm{N}-\mathrm{O}-\mathrm{S}$ atoms (Wacker and Pratto, 1964). Most of these lie in the range $1 / \lambda=10^{-1}$ to $10^{1} \mathrm{~cm}^{-1}$ and a few below $10^{-1} \mathrm{~cm}^{-1}$. At very low temperatures more of the molecules will condense on grains and their ice mantles will grow. Absorption from some of the largest grains will approach the peak of the weighting functions, but the microwave transitions of the few remaining volatile molecules will still be important to the Rosseland opacity.

At higher temperatures (up to about $20000 \mathrm{~K}$ ) electronic transitions in the molecules dominate the opacity. Much of the basic work on these transitions has been carried out by many investigators, but the application to high temperature molecular opacities was pioneered by Gilmore $(1965,1967)$, Armstrong and Nicholls (1967), Avilova et al. (1969a, b), Johnston et al. (1972), and Generosa and Harris (1973). The theories and calculations of all of these apply to air, however, they take into account the continuity and coexistence of molecules, atoms, and ions. Although some astrophysical molecular opacities had been calculated earlier (e.g., Tsuji, 1964, 1971; Auman and Bodenheimer, 1967; Linsky, 1969; Carbon et al., 1969; Alexander et al., 1971), efforts to preserve continuity and coexistence of atoms and molecules in astrophysical opacities are just getting started (Merts and Magee, 1973). The main reason for the delays in molecular opacity calculations are the lack of basic data and the difficulties encountered in obtaining them. Data for absolute measurements exist only for room temperature absorption bands, i.e., for transitions involving the vibrational level $v^{\prime \prime}=0$ in the electronic ground state. Most absorption data for excited states is deduced from emission measurements or from calculations. Unfortunately emission usually involves transitions between many closely spaced initial and final states, making analysis difficult and often impossible. Calculated results often suffer because a multiconfiguration approximation may be required but is too complex and therefore 
not used. The $\mathrm{N}_{2}$ Birge-Hopfield band system is a typical example of the complexities encountered in relatively simple molecules. Figure 2 illustrates how, in the energy range from about 12.5 to $14 \mathrm{eV}$ above the ground state of $\mathrm{N}_{2}$, the unperturbed potential curves belonging to pairs of states with the same symmetry character intersect. Near the intersections the superposition of these states is the cause for strong perturbations altering the transition probabilities and destroying the otherwise regular spacing of the vibrational levels. One pair of intersecting states has been labeled $b^{1} \Pi_{u}$ and $c^{1} \Pi_{u}$, the other one $b^{1} \Sigma_{u}^{+}$and $c^{1} \Sigma_{u}^{+}$. The Birge-Hopfield band systems 1 and 2 are associated with the valence states $b^{\prime}$ and $b$, respectively. States $c^{\prime}$ and $c$ are Rydberg states. The proper identification of these levels succeeded only relatively recently

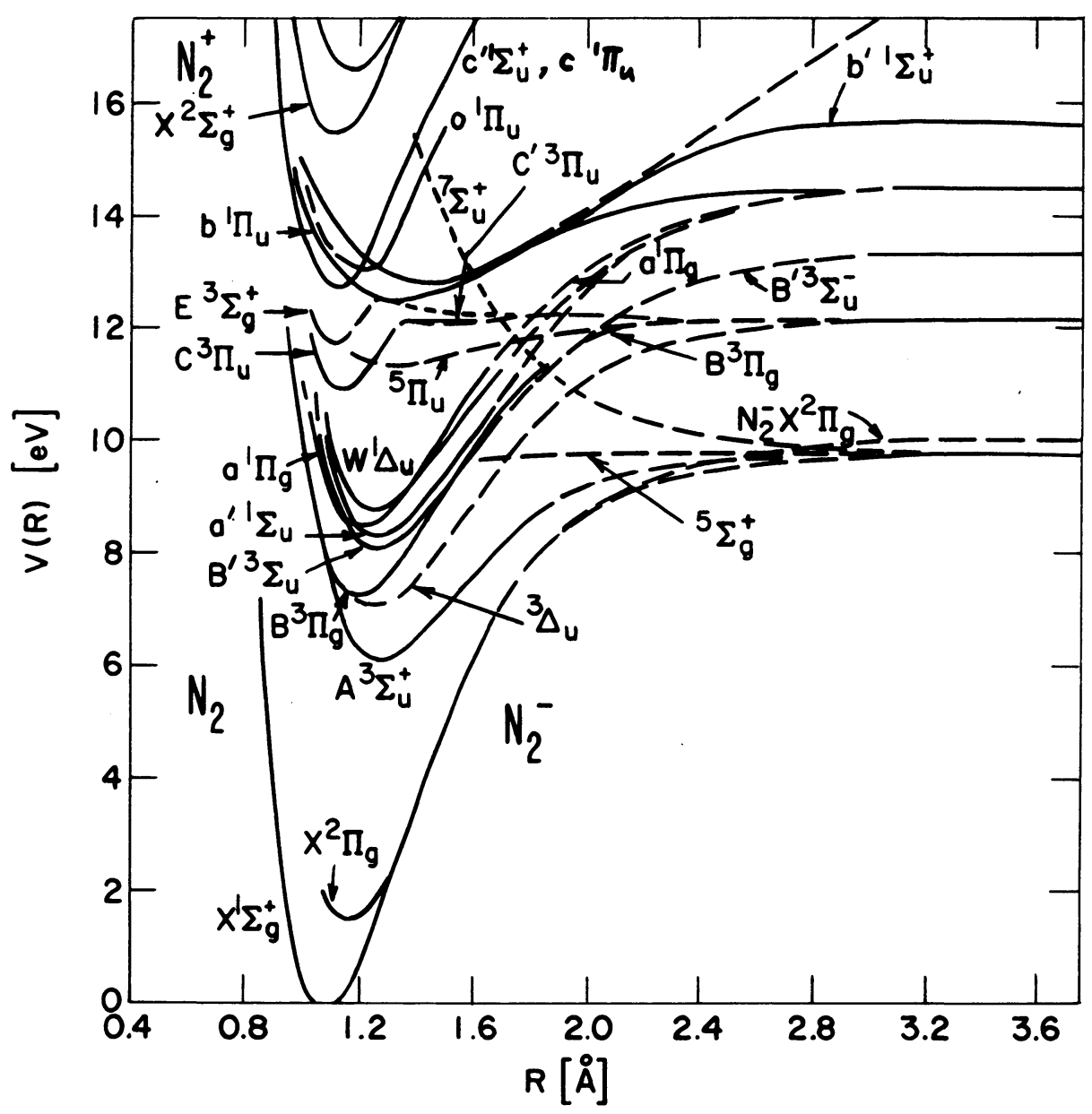

Fig. 2. Potential energy curves for nitrogen (taken from Gilmore, 1967) showing the intersection of $b, b^{\prime}, c$, and $c^{\prime}$. Curves $c$ and $c^{\prime}$ are drawn as one curve. The separated atom states at large $R$ from top to bottom correspond to $\mathrm{N}\left({ }^{2} D^{\circ}\right)+\mathrm{N}\left({ }^{2} P^{\circ}\right), \mathrm{N}\left({ }^{2} D^{\circ}\right)+\mathrm{N}\left({ }^{2} D^{\circ}\right), \mathrm{N}\left({ }^{4} S^{\circ}\right)+\mathrm{N}\left({ }^{2} P^{\circ}\right), \mathrm{N}\left({ }^{4} S^{\circ}+\mathrm{N}\left({ }^{2} D^{\circ}\right)\right.$, $\mathrm{N}\left({ }^{4} S^{\circ}\right)+\mathrm{N}^{-}\left({ }^{3} P\right)$, and $\mathrm{N}\left({ }^{4} S^{\circ}\right)+\mathrm{N}\left({ }^{4} S^{\circ}\right) .\left(1 \mathrm{eV} \equiv 0.8066 \mu \mathrm{m}^{-1}\right)$. 
(Dressler, 1969) and is a necessary prerequisite for determining Franck-Condon factors and for making theoretical predictions of the band absorption. Similar diffculties exist with many other simple molecules.

\section{Status of Opacity Calculations}

Two basic sets of information are needed for opacity calculations: the equation of state of the medium and the radiative cross sections.

The equation of state is determined by the equilibrium between ions (and electrons), atoms, molecules, and molecular ions (all in the gaseous phase), and condensed matter.

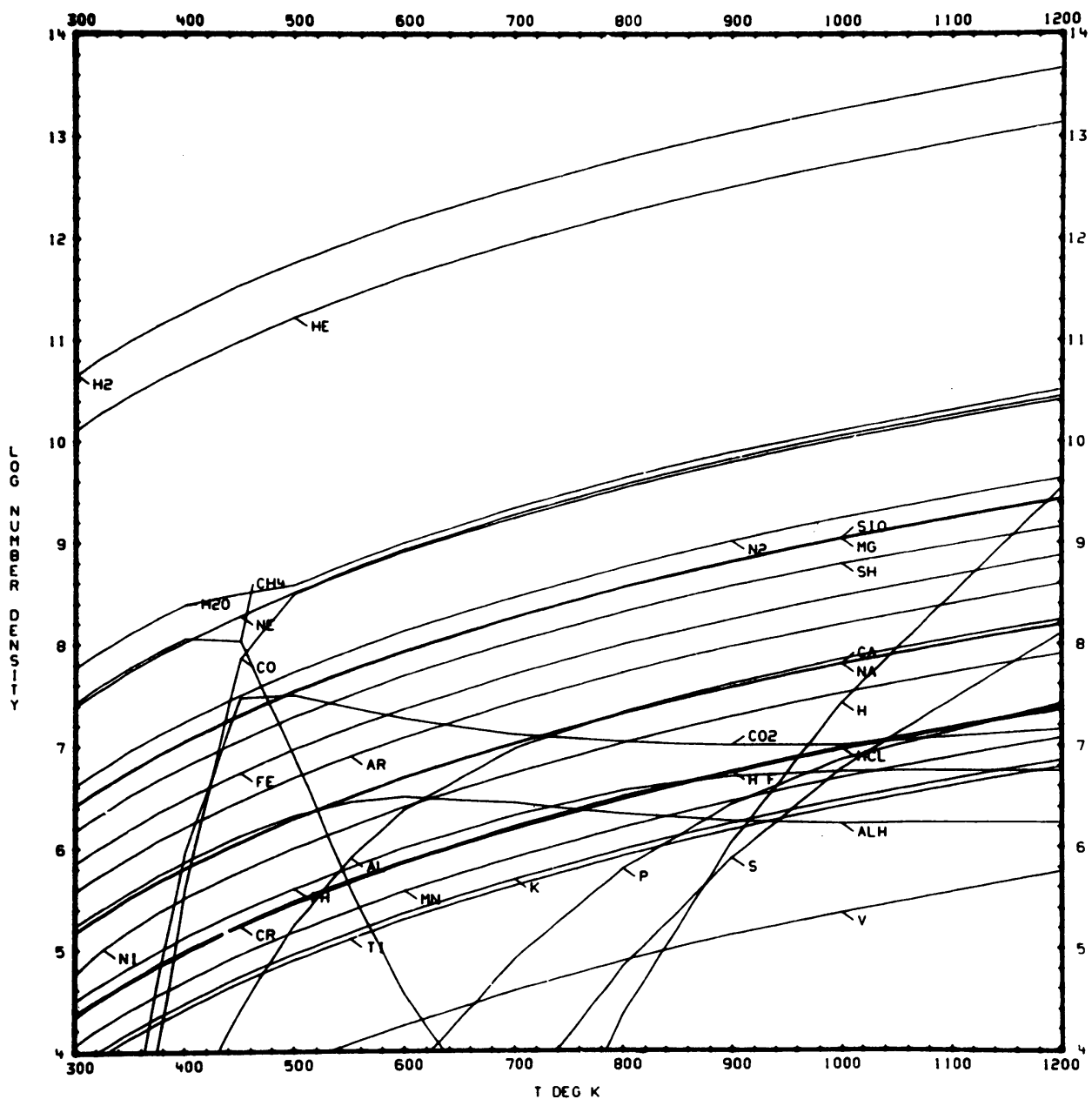

Fig. 3. Chemical abundances (without condensation) in proto-solar nebula assuming $Q=\left(T \mathrm{~K} / 10^{5}\right)^{5}$ $\mathrm{g} \mathrm{cm}^{-3}$. For clarity only a limited temperature range is shown. $\mathrm{NH}_{3}$ increases very rapidly just below $300 \mathrm{~K}$. Above $12000 \mathrm{~K}$ a few more diatomics (primarily $\mathrm{OH}$ and $\mathrm{MgH}$ ) contribute significantly.

Standard astrophysical abundances were assumed for the elements. 
Programs to compute the equilibrium in the gaseous phase alone - i.e., the chemistry, see, e.g., Figure 3, - have been available for some time. Only very limited efforts have been made to include the condensed phase of matter in the equilibrium (Duff, 1962; Tsuji, 1966).

The collective absorption and scattering by particles (grains as well as liquid drops) also requires knowledge of the particle size distributions. Usually Gaussian or powerlaw distributions with cutoffs are adopted.

The physics for commencement of condensation in the microscopic realm is insufficiently understood. It is not clear whether a better understanding of this process would shed more light on particle distributions. The absorption and scattering of radiation by 'grains' is well developed in the Mie theory. Programs to compute the cross sections for 2-component refractory core-ice mantle spheres using complex and wavelength-dependent refractive indices are available (e.g., see Figure 1). Similar programs for spheroidal particles could be developed. Some parameters for the geometric asymmetry could be obtained, e.g., from observed polarization of zodiacal light (Greenberg, 1970).

Data for microwave transitions and infrared rotation-vibration absorption is available, but more data will be needed. Data for electronic transitions involving highly excited states of molecules are considerably more sparse and unreliable. There are no difficulties with the atomic-ionic cross sections of astrophysical interest that could not be resolved with presently available methods and programs.

\section{Acknowledgement}

Our gratitude goes to R. K. M. Landshoff, who provided insight and valuable discussions on molecular transitions.

\section{References}

Alexander, D., Collins, J., Fay, T., and Johnson, H. R.: 1971, Bull. Am. Astron. Soc. 3, 380.

Anders, E.: 1968, Accounts Chem. Res. 1, 289.

Anders, E.: 1971, Ann. Rev. Astron. Astrophys.9, 1.

Armstrong, B. H. and Nicholls, R. W.: 1967, 'Thermal Radiation Phenomena, Vol. 2, The Equilibrium Radiative Properties of Air-Theory', Lockheed Missiles and Space Company report LMSC3-27-67-1.

Auman, J. A. and Bodenheimer, P.: 1967, Astrophys.J. 149, 641.

Avilova, I. V., Biberman. L. M., Vorobjev, V. S., Zamalin, V. M., Kobzev, G. A., Lagar'kov, A. N., Mnatsakanian, A. Ch., and Norman, G. E.: 1969a, J. Quant. Spectrosc. Radiat. Transfer 9, 89.

Avilova, I. V., Biberman, L. M., Vorobjev, V. S., Zamalin, V. M., Kobzev, G. A., Lagar'kov, A. N., Mnatsakanian, A.Ch., and Norman, G. E.: 1969b, J. Quant. Spectrosc. Radiat. Transfer 9, 113. Carbon, D., Gingerich, O. J., and Latham, D. W.: 1969, in S. S. Kumar (ed.), Low Luminosity Stars, Gordon and Breach Science Publishers, New York, p. 435.

Cox, A. N. and Stewart, J. N.: 1965, Astrophys. J. Suppl. 11, 22.

Dressler, K.: 1969, Can. J.Phys. 47, 547.

Duff, R. E. and Bauer, S. H.: 1962, J. Chem. Phys. 36, 1754.

Gaustad, J. E.: 1963, Astrophys.J. 138, 1050.

Generosa, J. I. and Harris, R. A.: 1973, Air Force Weapons Laboratory, Kirtland AFB, N.M., private communication. 
Gilmore, F. R.: 1965, J. Quant. Spectrosc. Radiat.Transfer 5, 125.

Gilmore, F. R.: 1967, 'Thermal Radiation Phenomena, Vol. 1, The Equilibrium Radiative Properties of Air', Lockheed Missiles and Space Company report LMSC-3-27-67-1.

Greenberg, J. M.: 1970, in T. M. Donahue, P. A. Smith, and L. Thomas (eds.), Space Research X, North-Holland Publishing Company-Holland, p. 225.

Hayashi, C. and Nakano, T.: 1965, Progr. Theor. Phys. 34, 764.

Hayatsu, R., Studier, M. H., Oda, A., Fuse, K., and Anders, E.: 1968, Geochim. Cosmochim. Actn 32, 175.

Huffman, D. R. and Stapp, J. L.: 1971, Nature Phys. Sci. 229, 45.

Johnston, R. R., Landshoff, R. K. M., and Platas, O. R.: 1972, 'Radiative Properties of High Temperature Air', Lockheed Missiles and Space Company report LMSC D 267205.

Larimer, J. W.: 1967, Geochim. Cosmochim. Acta 31, 1215.

Larimer, J. W. and Anders, E.: 1967, Geochim. Cosmochim. Acta 31, 1239.

Linsky, J. L.: 1969, Astrophys. J. 156, 989.

Merts, A. L. and Magee, N. H.: 1973, Bull. Am. Astron. Soc. 5, 338.

Studier, M. H., Hayatsu, R., and Anders, E.: 1968, Geochim. Cosmochim. Acta 32151.

Tsuji, T.: 1964, Proc. Japan Acad.40, 99.

Tsuji, T.: 1966, Publ. Astron. Soc. Japan 18, 127; corrected: 1967, Publ. Astron. Soc. Japan 19, 119.

Tsuji, T.: 1971, Publ. Astron. Soc. Japan 23, 553.

Wacker, P. F. and Pratto, M. R.: 1964, 'Spectral Tables, Line Strength of Asymmetric Rotors', NBS Monograph 70 - Vol. II, U.S. Government Printing Office, Washington, D. C. 